\title{
A rare case of suicidal ideation related to Adalimumab use
}

Fatima Jafri

Amanda Sammut

Department of Medicine, Division of Rheumatology, Harlem Hospital Center, Columbia University Medical Center, New York, NY, USA
Correspondence: Fatima Jafri Department of Medicine, Harlem Hospital Center, 506 Lenox Avenue, New York, NY 10037, USA

Tel +0I 2129391506

Fax +0I 2129392263

Email jafrif@nychhc.org
This article was published in the following Dove Press journal: Open Access Rheumatology: Research and Reviews

Introduction: Patients undergoing treatment with an anti-tumor necrosis factor-alpha (TNF- $\alpha$ ) agent can, as an adverse event, develop anti-TNF- $\alpha$-induced lupus (ATIL). Neuropsychiatric symptoms such as depression and suicidal ideations are not commonly seen in patients who develop ATIL. We describe a case of a 56-year-old female who developed ATIL and suicidal ideations while on Adalimumab.

Case presentation: A 56-year-old female with rheumatoid arthritis (RA) and no known prior history of systemic lupus erythematosus or depression presented with suicidal ideations, joint pains and a malar rash after a recent change in her Adalimumab dose. She was treated for an acute ATIL episode based on her symptoms and serologies which were positive for anti-doublestranded deoxyribonucleic acid antibody. An inpatient psychiatric consultation determined that the patient's suicidal ideations may be an associated symptom of her current ATIL episode or possibly secondary to her chronic pain and debilitation from her RA. The patient's Adalimumab was discontinued and she was treated with a course of intravenous glucocorticoid. The patient's suicidal ideations resolved and her anti-double-stranded deoxyribonucleic acid antibody serologies became negative. She was subsequently started on Abatacept and has achieved remission of her RA with no further suicidal ideations.

Discussion: The development of ATIL in patients undergoing treatment with an anti-TNF- $\alpha$ agent is a rare occurrence. The aim of reporting our case is to increase understanding of ATIL by highlighting the occurrence of neuropsychiatric symptoms in a patient who developed ATIL.

Keywords: Adalimumab, lupus, rheumatoid arthritis, suicidal ideation

\section{Introduction}

Adalimumab is currently approved for the treatment of rheumatoid arthritis (RA). ${ }^{1}$ A rare adverse event associated with the use of Adalimumab is the development of antitumor necrosis factor-alpha (anti-TNF- $\alpha$ )-induced lupus (ATIL). ${ }^{2}$ ATIL has been described as the development of the syndrome of drug-induced lupus secondary to an anti-TNF- $\alpha$ agent. ${ }^{3}$ While the occurrence of autoantibodies is not uncommonly noted in patients treated with anti-TNF- $\alpha$ agents, what is uncommon is the development of lupus-like symptoms. ${ }^{4}$ Furthermore, the relationship between the presence of neuropsychiatric symptoms in patients who have developed ATIL is not well understood, given the paucity of reported ATIL cases in the literature and the probable overall challenging nature in identifying neuropsychiatric symptoms. As of now, depression and suicide are not significantly known to be direct adverse events associated with Adalimumab use. ${ }^{5}$ The aim of reporting our case is to increase our understanding of 
ATIL by highlighting the occurrence of neuropsychiatric symptoms in a patient who developed ATIL. Informed written and verbal consent was obtained from the patient to permit us to publish her case for further scientific learning.

\section{Case report}

A 56-year-old female with a past medical history of hypertension and hyperlipidemia was diagnosed $\sim 15$ years ago with RA in Honduras. She first established care in our rheumatology clinic $\sim 4$ years ago. She endorsed ongoing symptoms of joint pains, joint tenderness and morning stiffness. Her outpatient physical exam was consistent with swelling, tenderness and limited mobility of multiple joints. Her labs were significant for an elevated level of anti-citrullinated peptide antibody, an elevated level of C-reactive protein and an elevated erythrocyte sedimentation rate. Her rheumatoid factor was negative. She also had radiographic evidence of bony erosions on X-ray. In addition, she was evaluated as not having a diagnosis of systemic lupus erythematosus (SLE). Her RA diagnosis met the 2010 American College of Rheumatology diagnostic criteria for the diagnosis of RA. ${ }^{6}$

The patient was initially treated with methotrexate, but after failure to achieve remission, methotrexate was stopped and she was started on Adalimumab at a dose of $40 \mathrm{mg}$ subcutaneously every 2 weeks. She continued to have recurrent symptoms of joint pain and her dose of Adalimumab was subsequently increased to Adalimumab $40 \mathrm{mg}$ subcutaneously every week. Approximately 2 weeks after increasing her dose of Adalimumab, she presented to the rheumatology clinic with a facial rash, oral ulcers, bilateral shoulder pain, bilateral knee pain and suicidal ideations. She was sent to the emergency room for further evaluation. She described no previous suicidal ideations or suicidal attempts and stated that she started to develop suicidal ideations over the last few days. On presentation, she was tachycardic with a heart rate of 108 beats per minute and afebrile. Her examination was notable for a facial rash, oral ulcers, mild bilateral shoulder and bilateral knee pain on palpation. Her laboratory results were significant for an elevated anti-nuclear antibody titer of $1: 1,280$ (normal is $<1: 80$ ), a strongly positive anti-histone antibody level of 2.6 (normal is $<1$ Unit) and a borderline positive anti-double-stranded DNA antibody (anti-dsDNA ab) level of $33 \mathrm{IU} / \mathrm{mL}$ (normal is $<30 \mathrm{IU} / \mathrm{mL}$ ). She was admitted for an ATIL episode and a psychiatric consultation. The assessment made by the psychiatric consultation team was that while the patient had suicidal ideations, there was no history of a current plan for suicide and her suicidal ideations might be an associated symptom of her current ATIL episode or possibly secondary to her chronic pain and debilitation from her RA. The recommendation from the psychiatry team was close follow-up with better control of the patient's RA symptoms and to continue to treat the active ATIL episode.

The patient's Adalimumab was stopped at the start of her hospitalization and she was treated with a course of intravenous glucocorticoid. Her joint pains resolved, rashes improved and she was no longer having suicidal ideations. She was subsequently started on Abatacept and closely followed up in the outpatient setting. After a few weeks, her anti-dsDNA abs were repeated and were negative. Remission of her RA was also gradually achieved with no further episodes of suicidal ideations.

\section{Discussion}

Most cases of ATIL are diagnosed when there is a temporal relationship between the development of lupus-like symptoms and current use of an anti-TNF- $\alpha$ agent. ${ }^{7.8}$ In addition, there should also be the presence of serologic American College of Rheumatology criteria of SLE and no known previous history of SLE. The most common symptoms of ATIL are occurrence of a rash, joint pain and fever. ${ }^{9}$ The estimated incidence of ATIL is $0.19 \%-0.22 \%$ for infliximab, $0.18 \%$ for etanercept and $0.10 \%$ for Adalimumab. ${ }^{9}$ Neuropsychiatric features are not a common occurrence in ATIL. ${ }^{10}$ This is one case in which the patient had associated suicidal ideations with ATIL. It is, however, not possible based on this case to prove a causal relationship of Adalimumab use and suicidal ideation. It is possible that this patient was at risk for ATIL and that she had suicidal ideations as a rare manifestation of ATIL. A limitation in this case is that neither there was any objective measure used to assess for depression or suicidal ideations, such as use of the Beck Depression Inventory or Beck Suicidal Ideation Scale, when the patient presented with suicidal ideations, nor did we use any objective measures to reassess symptoms on follow-up. We suggest that it may be beneficial for future consideration to screen patients with ATIL for neuropsychiatric manifestations using objective measures to better understand their rate of occurrence.

\section{Disclosure}

The authors report no conflicts of interest in this work.

\section{References}

1. Navarro-Sarabia F, Ariza-Ariza R, Hernández-Cruz B, Villanueva I. Adalimumab for treating rheumatoid arthritis. $J$ Rheumatol. 2006;33(6):1075-1081.

2. Ramos-Casals M, Brito-Zerón P, Muñoz S, et al. Autoimmune diseases induced by TNF-targeted therapies: analysis of 233 cases. Medicine. 2007;86(4):242-251. 
3. Debandt M, Vittecoq O, Descamps V, Le Loët X, Meyer O. Anti-TNF alpha-induced systemic lupus syndrome. Clin Rheumatol. 2003;22:56-61.

4. Kang MJ, Lee YH, Lee J. Etanercept-induced systemic lupus erythematosus in a patient with rheumatoid arthritis. J Korean Med Sci. 2006;21(5):946-949.

5. Scheinfeld N. Adalimumab: a review of side effects. Expert Opin Drug Saf. 2005;4(4):637-641.

6. Aletaha D, Neogi T, Silman AJ, et al. 2010 Rheumatoid arthritis classification criteria: an American College of Rheumatology/European League Against Rheumatism collaborative initiative. Arthritis Rheum. 2010;62(9):2569-2581.
7. De Bandt M, Sibilia J, Le Loët X, et al. Systemic lupus erythematosus induced by anti-tumour necrosis factor alpha therapy: a French national survey. Arthritis Res Ther. 2005;7(3):R545-R551.

8. Ramos-Casals M, Brito-Zerón P, Soto MJ, Cuadrado MJ, Khamashta MA. Autoimmune diseases induced by TNF-targeted therapies. Best Pract Res Clin Rheumatol. 2008;22(5):847-861.

9. Almoallim H, Al-Ghamdi Y, Almaghrabi H, Alyasi O. Anti-tumor necrosis factor- $\alpha$ induced systemic lupus erythematosus. Open Rheumatol J. 2012;6:315-319.

10. Williams EL, Gadola S, Edwards CJ. Anti-TNF-induced lupus. Rheumatology. 2009;48(7):716-720.
Open Access Rheumatology: Research and Reviews

\section{Publish your work in this journal}

Open Access Rheumatology: Research and Reviews is an international, peerreviewed, open access journal publishing original research, reports, editorials, reviews and commentaries on all aspects of clinical and experimental rheumatology in the clinic and laboratory including the following topics: Pathology, pathophysiology of rheumatological diseases; Investigation, treatment and

\section{Dovepress}

management of rheumatological diseases; Clinical trials and novel pharmacologi$\mathrm{cal}$ approaches for the treatment of rheumatological disorders. The manuscript management system is completely online and includes a very quick and fair peer-review system, which is all easy to use. Visit http://www.dovepress.com/ testimonials.php to read real quotes from published authors. 